\title{
A Simple Microscopy Assay to Teach the Processes of Phagocytosis and Exocytosis
}

\author{
Ross Gray, ${ }^{*}$ Andrew Gray, Jessica L. Fite, Renée Jordan, Sarah Stark, \\ and Kari Naylor
}

Department of Biology, University of Central Arkansas, Conway, AR 72034

Submitted July 25, 2011; Revised January 17, 2012; Accepted January 22, 2012

Monitoring Editor: Mary Lee S. Ledbetter

\begin{abstract}
Phagocytosis and exocytosis are two cellular processes involving membrane dynamics. While it is easy to understand the purpose of these processes, it can be extremely difficult for students to comprehend the actual mechanisms. As membrane dynamics play a significant role in many cellular processes ranging from cell signaling to cell division to organelle renewal and maintenance, we felt that we needed to do a better job of teaching these types of processes. Thus, we developed a classroombased protocol to simultaneously study phagocytosis and exocytosis in Tetrahymena pyriformis. In this paper, we present our results demonstrating that our undergraduate classroom experiment delivers results comparable with those acquired in a professional research laboratory. In addition, students performing the experiment do learn the mechanisms of phagocytosis and exocytosis. Finally, we demonstrate a mathematical exercise to help the students apply their data to the cell. Ultimately, this assay sets the stage for future inquiry-based experiments, in which the students develop their own experimental questions and delve deeper into the mechanisms of phagocytosis and exocytosis.
\end{abstract}

\section{INTRODUCTION}

Phagocytosis, a specialized form of endocytosis, is one method of ingesting extracellular material, while exocytosis is the process in which intracellular vesicles fuse with the plasma membrane and release, or secrete, their contents into the extracellular space (Alberts et al., 2008). These processes not only are critical to the cell, but are also critical for students to understand. Phagocytosis and exocytosis fall into a category of cellular reactions that require membrane dynamics. These reactions also require significant contribution by the cytoskeleton. By understanding the mechanisms behind phagocytosis and exocytosis, students are better able to understand the myriad other cellular processes that re-

DOI: $10.1187 /$ cbe.11-07-0060

*These authors contributed equally to this work.

Address correspondence to: Kari Naylor (kknaylor@uca.edu).

(C) 2012 R. Gray et al. CBE-Life Sciences Education (c) 2012 The American Society for Cell Biology. This article is distributed by The American Society for Cell Biology under license from the author(s). It is available to the public under an AttributionNoncommercial-Share Alike 3.0 Unported Creative Commons License (http://creativecommons.org/licenses/by-nc-sa/3.0).

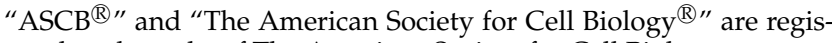
tered trademarks of The American Society for Cell Biology. quire membrane dynamics and/or the cytoskeleton, such as cell signaling, organelle maintenance, and vesicle trafficking. Surprisingly, as important as these processes are, in many cell biology courses very few of the molecular mechanisms are discussed. In fact, the subjects of phagocytosis and exocytosis are often only quickly covered near the end of the semester, leading to a poor understanding of both processes and membrane dynamics as a whole. Even when these subjects are covered in better detail, many students find it hard to grasp the whole context of membrane fusion, vesicle formation, and molecule transport in a lecture setting. To fully appreciate these processes, many students need to see them occurring in living cells. This can be especially important for students who are predominantly sensing, visual, inductive, active, and global learners, as these students rarely get their educational needs met in the classical lecture-style science courses (Felder, 1993).

The teaching style in most lecture courses is often biased toward the college students who are intuitive, verbal, reflective, and sequential learners (Felder, 1993). This imbalance puts a sizable fraction of the student population at a disadvantage. Laboratory courses, being inherently sensory, visual, and active should, in principle, compensate for a portion of this imbalance (Felder, 1993). We therefore designed a phagocytosis/exocytosis laboratory experiment for our cell biology course. Cell biology at the University of Central Arkansas 
is a combined lecture and laboratory course for juniors and seniors. It is required for all students obtaining a biology degree; this includes students with an emphasis in ecology/organismal courses and students with an emphasis in molecular/cellular-based courses.

Inquiry-based experiments, when properly presented by faculty and engaged in by students, improve analytical skills, increase students' enthusiasm for science, and can more effectively teach science content than lecture alone (Olson and Loucks-Horsley, 2000; Gibson and Chase, 2002). Thus, we designed this experiment to be an inquiry-based one. In its entirety, this experiment requires a minimum of two 3-h laboratory periods, but can easily span three to four laboratory sessions. In this paper, we describe a novel method to simultaneously quantify phagocytosis and exocytosis in Tetrahymena. This is performed in the first laboratory period and is used to enhance the experience in subsequent laboratory sessions, the inquiry-based labs, in which the students identify experimental questions to be asked about the mechanisms of phagocytosis and/or exocytosis. In addition, we present our assessment of student learning as a measure of the assay's effectiveness as a teaching tool. Our goals in this exercise are twofold: 1) to teach the processes of phagocytosis and exocytosis, emphasizing the function of the cytoskeleton, and 2) to set the stage for future inquiry-based classroom experiments.

For this experiment we used the model organism Tetrahymena pyriformis, a freshwater, ciliated protozoan that is easily obtained (Carolina Biological, Burlington, NC). Tetrahymena is an ideal candidate for the study of macro-level cellular processes by students, because phagocytosis and exocytosis are readily observable with a compound microscope. When hungry, Tetrahymena use their cilia to sweep particles into their oral groove and then create a food vacuole via phagocytosis. The food vacuole is subsequently transported to the lysosome by the action of actin filaments (it is unclear whether microtubules are involved at this stage). This process can be visualized by feeding the cells particles of India ink. The rate of phagocytosis is quantified by counting the vesicles that form within Tetrahymena in a defined time period (Bozzone, 1999). This process can also be visualized by feeding the cells carmine dye, which results in red vesicles.

Exocytosis in Tetrahymena occurs by a process called egestion. During egestion, the food vacuoles, now containing indigestible molecules, leave the lysosome and travel to the cytoproct, the site of egestion. It is thought that microtubules may function in guiding the food vacuoles to the cytoproct, at which point the food vacuole membrane fuses with the plasma membrane to create a fusion pore. The contents of the food vacuole are released to the exterior of the cell as the fusion pore widens. The vacuole is ultimately resorbed into the cytoplasm by endocytosis at the cytoproct, through a process known as membrane recycling (Rothstein and Blum, 1974; Nilsson, 1977; Allen and Wolf, 1979; Ricketts, 1979; Fok and Shockley, 1985; Sugita et al., 2009). The process of egestion in Tetrahymena is easily visualized with a compound microscope by feeding the cells India ink and monitoring the loss of India ink-stained vesicles over time (Tiedtke et al., 1988).

In our classroom experiment, we developed a method for students to simultaneously quantify phagocytosis and egestion by counting the vesicles entering (phagocytosis) or exiting (egestion) the cell as a function of time. Additionally, we explored the function of the cytoskeleton in phagocytosis and exocytosis with the actin inhibitor, latrunculin A (Lat-A), and the microtubule inhibitor, colchicine.

Understanding the processes of phagocytosis and exocytosis provides a solid foundation for understanding other important cellular processes, such as secretion and cell signaling, which also depend on membrane dynamics. By presenting this information in a laboratory setting, we can appeal to students who are better visual, sensory, and active learners (Felder, 1993). Additionally, our protocol provides a framework for a variety of future inquiry-based experiments, such as having the students test the effect of blocking lysosomal fusion on the processes of phagocytosis and exocytosis. On the basis of our assessments, we found that students showed a positive response in their understanding of phagocytosis and exocytosis and learned a critical application of biological mathematics. Mastery of both concepts can serve as important stepping-stones to future inquiry-based learning exercises.

\section{MATERIALS AND METHODS}

\section{Measuring Phagocytosis and Exocytosis}

Phagocytosis and exocytosis were measured simultaneously using a simple India ink and carmine dye assay. For a detailed protocol, please see Supplemental Material 1. All procedures were carried out at room temperature. In brief, Tetrahymena cells were cultured for $3-4 \mathrm{~d}$ in $2 \%$ proteose peptone, and a small aliquot of these cells was incubated with an equal volume of $1 \%$ India ink (Hunt Speedball; Hobby Lobby, Conway, AR) in distilled water for $30 \mathrm{~min}$. Cells were washed twice by centrifuging for $3 \mathrm{~min}$ at $200 \times g$. Carmine (C1022; Sigma-Aldrich, St. Louis, MO) was prepared by suspending $20 \mathrm{mg} / \mathrm{ml}$ of carmine in distilled water, which was followed by vortexing for $1 \mathrm{~min}$ and centrifuging for $3 \mathrm{~min}$ at $600 \times g$. The supernatant was used for the experiment (Blum and Greenside, 1976). Carmine was added to the washed cells at $0.1 \mathrm{ml}$ of carmine per $0.5 \mathrm{ml}$ of cells. Cells were analyzed by counting the red and black vesicles at 10,20 , and 30 min with a $10 \times$ objective. For drug treatment, the cells were exposed to the drugs after the India ink was washed away, but before introduction of carmine. Lat-A ( L5163; SigmaAldrich) was resuspended at $100 \mu \mathrm{g} / \mathrm{ml}$ in ethanol (EtOH) and used at $0.2 \mu \mathrm{g} / \mathrm{ml}$. An equal volume of $\mathrm{EtOH}$ was used as the control. Colchicine (C9754; Sigma-Aldrich) was resuspended at $10 \mathrm{mg} / \mathrm{ml}$ in distilled water (alternatively, media can be used) and used at $4 \mathrm{mg} / \mathrm{ml}$. An equal volume of distilled water was used as a control. Cells were incubated for $10 \mathrm{~min}$ with respective treatments, and then carmine was added directly to the reaction.

\section{Data Collection}

All stained vesicles were counted for 60 cells for each condition at each time point. Vesicle number ranged from 3-10 vesicles per cell, with a few cells having as many as 20 vesicles. Cells with no stained vesicles typically had just completed cell division and had yet to fully reassemble their oral grooves, which temporarily inhibited phagocytosis. 


\section{Experimental Data Analysis}

We assessed phagocytosis by the increase in red/carmine vesicles over time. Egestion was analyzed by the decrease in black/India ink vesicles over time. Data are presented as average number of vesicles (red/black)/cell with SE. Statistical analysis was performed by two-way analysis of variance and Tukey HSD post hoc test using JMP 9.0.0 (SAS Institute, Cary, NC) software.

All student assessments were deemed exempt by the University of Central Arkansas Institutional Review Board council.

\section{RESULTS}

\section{Effect of Actin Cytoskeleton on Phagocytosis and Exocytosis}

When we designed our teaching lab experiment to teach the effects of the cytoskeleton on phagocytosis and exocytosis, we wanted to ensure that we were creating a professional research environment in the classroom. Thus, we first determined the effect of cytoskeletal inhibitors on exocytosis and phagocytosis in T. pyriformis to determine whether our classroom-based protocol would give results comparable with those found in the literature.

To assess the effect of cytoskeletal inhibitors on phagocytosis and exocytosis, we measured vesicle formation over time in the presence and absence of cytoskeletal drugs. T. pyriformis cells were incubated in India ink, washed twice, and then incubated in carmine dye. Vesicles containing India ink appeared black under a compound microscope. The number of black vesicles decreased over time and served as a measure of exocytosis, while vesicles containing carmine dye appeared red, increased over time, and were used to measure phagocytosis (Supplemental Material 1).

We investigated the function of actin in phagocytosis by quantifying the increase in vesicle formation over time in the presence and absence of Lat-A, an actin polymerization inhibitor (Coué et al., 1987; Morton et al., 2000). Statistical analysis of the time effect showed that phagocytosis was occurring with $(p=0.0013)$ and without $(p<0.0001)$ Lat-A (Figure 1$)$. More importantly, our results demonstrated that the number of vesicles formed over time was significantly decreased in the presence of Lat-A ( $p<0.0001)$, as compared with the EtOH control (Figure 1); thus, Lat-A decreased phagocytosis.

To determine whether actin functions in exocytic egestion, we assessed the decrease in vesicle number over time. As expected, time had an effect with and without Lat-A (LatA, $p=0.0005$; control, $p=0.0053$ ), indicating that vesicle number changed over time (Figure 2 ), and the presence of Lat-A did have a significant inhibitory effect on egestion $(p=$ 0.0002; Figure 2). Thus, actin, which is necessary for phagocytosis, (Rothstein and Blum, 1974; Bozzone, 1999) is also necessary for exocytosis, as has been suggested by Allen and Wolf (1979).

\section{Effect of Microtubules on Phagocytosis and Exocytosis}

Colchicine, an inhibitor of microtubule polymerization, was used to study the effect of microtubules on phagocytosis and egestion. During phagocytosis, time significantly affected the
Phagocytos is: Actin

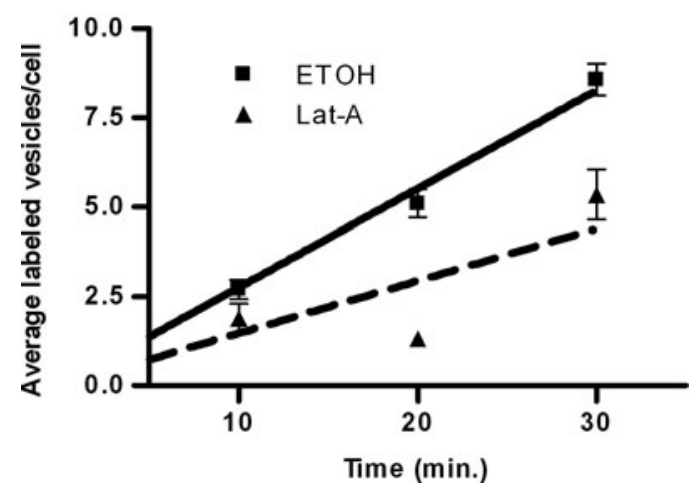

Figure 1. Effect of Lat-A on the increase in vesicle number per cell. The data indicate that actin is necessary for phagocytosis. The solid line represents control cells incubated with EtOH; the dashed line indicates treated cells incubated with $0.2 \mu \mathrm{g} / \mathrm{ml}$ Lat-A. Values are mean vesicle number per cell \pm SE of 60 cells for each time point. Trend lines were determined by nonlinear regression analysis.

vesicle number in the presence $(p<0.0001)$ and absence $(p<$ 0.0001) of colchicine, indicating that phagocytosis was taking place (Figure 3). Colchicine significantly decreased the number of vesicles formed by phagocytic ingestion of carmine dye $(p<0.0001)$, indicating that microtubules contribute to phagocytosis (Figure 3).

Previous studies have shown that microtubules in Tetrahymena are present at the cytoproct, the site of egestion, although their function is poorly defined (Allen and Wolf, 1974, 1979; Kovacs and Csaba, 2006; Sugita et al., 2009). Our data suggest that while egestion was slower with colchicine (time did not have a significant effect, $p=0.3554)$ than without $(p=0.0063)$, there was no significant difference in the rates $(p=0.4840)$ (Figure 4).

In conclusion, our results from our teaching assay, which simultaneously measures phagocytosis and exocytosis in Tetrahymena, confirm that microtubules are important to the

Egestion: Actin

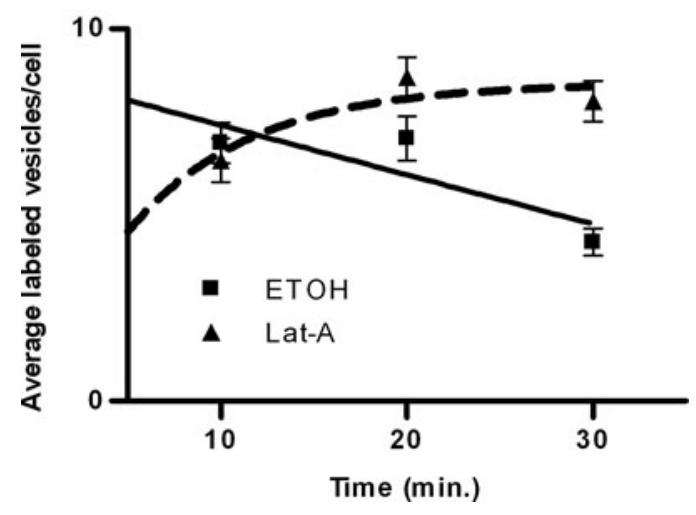

Figure 2. Effect of Lat-A on the decrease in vesicle number per cell. The data indicate that actin is necessary for egestion. The solid line represents control cells incubated with $\mathrm{EtOH}$; the dashed line indicates treated cells incubated with $0.2 \mu \mathrm{g} / \mathrm{ml}$ Lat-A. Values are mean vesicle number per cell $\pm S E$ of 60 cells for each time point. Trend lines were determined by nonlinear regression analysis. 


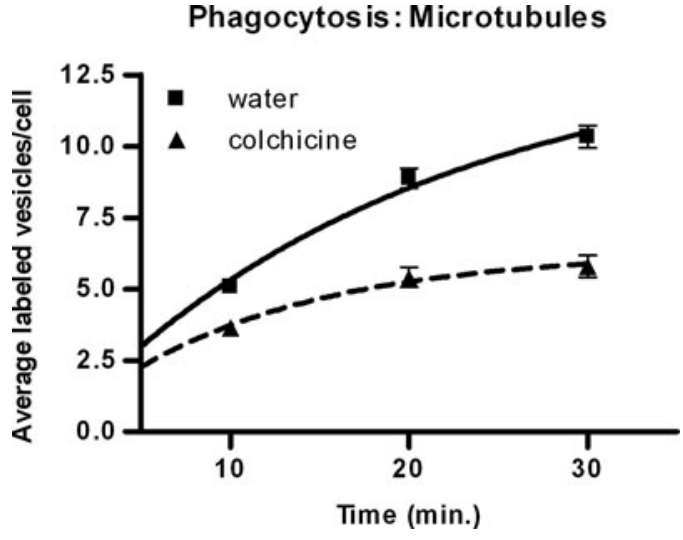

Figure 3. Effect of colchicine on the increase in vesicle number per cell. The data indicate that microtubules are necessary for phagocytosis. The solid line represents control cells incubated with water; the dashed line indicates treated cells incubated with $4 \mathrm{mg} / \mathrm{ml}$ colchicine. Values are mean vesicle number per cell \pm SE of 60 cells for each time point. Trend lines were determined by nonlinear regression analysis.

process of phagocytosis, as has been previously shown (Rothstein and Blum, 1974; Bozzone, 1999; Kovacs and Csaba, 2006), but it remains unclear to what extent the microtubules are involved in exocytic egestion.

\section{Assessment of Experiment as a Teaching Tool}

Once we demonstrated that our experimental design to simultaneously quantify phagocytosis and exocytosis produced results comparable with the published literature of individual experiments, we assessed whether it fulfilled our goals for student learning outcomes. We expected the students to learn about the molecular mechanisms of phagocytosis and exocytosis, with an emphasis on the function of the cytoskeleton. Therefore, we invited current cell biology students to attend an extra laboratory session. Thirty-one students attended and completed our learning assessment both prior to the experiment and after the experiment was com-

\section{Egestion: Microtubules}

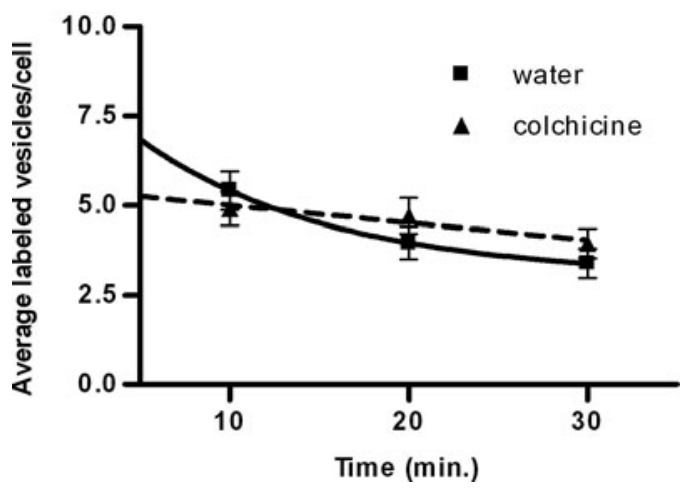

Figure 4. Effect of colchicine on the decrease in vesicle number per cell. The data indicate that colchicine does not significantly affect egestion. The solid line represents control cells incubated with water; the dashed line indicates treated cells incubated with $4 \mathrm{mg} / \mathrm{ml}$ colchicine. Values are mean vesicle number per cell $\pm S E$ of 60 cells for each time point. Trend lines were determined by nonlinear regression analysis. pleted. Before beginning the assay, a brief prelab discussion was held. We briefly discussed the organism, Tetrahymena, and explained the purpose of phagocytosis and exocytosis in Tetrahymena, as well as the major points of the protocol. The student assessment consisted of five questions pertaining to the process of exocytosis, vesicle pathways, and data analysis (Table 1 and Supplemental Material 2). The assessments were graded (points awarded were 0 for incorrect, 1 for partially correct, or 2 for a perfect answer), and the mean question score prior to the experiment was compared with the mean question score after the experiment (Table 1). Question 1 asked the students how to effectively measure the rate of exocytosis. The mean scores did not show a significant difference $(p=0.5552)$, with the average score preexperiment being 1.2258, while the postexperiment average was 1.3548. Our second question asked students whether the cytoskeleton is involved in exocytosis, and if so, what particular cellular structure is involved. The mean score after the experiment ( $x=1.09677)$ was significantly higher than before the experiment $(x=0.22581 ; p<0.0001)$. The answers to the third question, "Why do Tetrahymena egest?," showed no significant difference in the mean score after the experiment was performed (pre- $x=1.1629$, post- $x=1.38710$; $p=$ 0.3220 ). Question 4 asked students to describe the pathway of vesicles from ingestion to egestion. The mean score after the experiment $(x=1.70968)$ was significantly higher than before the experiment $(x=0.74194 ; p<0.0001)$. Our final question, number 5, asked students to correctly interpret graphed data. There was no significant difference in score means prior to $(x=1.16129)$ or after $(x=0.87097 ; p=0.2229)$ the experiment.

In summary, students were successfully able to answer questions about the effect(s) of the cytoskeleton on exocytosis (questions 2 and 4) after the laboratory experiment. Student answers to question 2 demonstrated that the students did learn about cytoskeletal effect(s) on the process of exocytosis, while their answers to question 4 showed that they learned about membrane dynamics and one vesicle-trafficking pathway in the cell. Questions 1 and 5 tested the students' ability in experimental design and data analysis, respectively. The results indicate that the students were capable of answering question 1 prior to the experiment, most probably because some of our students have dealt with rates in past experiments and were able to extrapolate this knowledge to answer our question. Interestingly, their scores for question 5 decreased after the assay, demonstrating that they were unsuccessful at analyzing hypothetical data. Finally, question 3 asked about the information presented in the presentation portion (prelab) of the experiment. Prior to the prelab discussion and experiment, the students were partially correct in their responses (mean $=1.1629$ ); no gains were seen in new understanding of the material that was strictly presented in a lecture format after the exercise.

\section{Membrane Turnover Exercise}

Difficulties with subject comprehension in lecture-based classes are not the only issue that science students face. We have observed over the years that students are quite capable with most qualitative and simple quantitative analyses but struggle when asked to apply calculations to biological systems. Thus, we developed an exercise to calculate membrane turnover in Tetrahymena (Supplemental Material 3). This 
Table 1. Average assessment scores before and after phagocytosis/exocytosis laboratory experiment ${ }^{\mathrm{a}}$

\begin{tabular}{|c|c|c|c|}
\hline Question & $\begin{array}{l}\text { Mean before } \\
\text { assessment }\end{array}$ & $\begin{array}{l}\text { Mean after } \\
\text { assessment }\end{array}$ & $p$ Value \\
\hline 1. How can you measure the rate of exocytosis? & 1.2258 & 1.3548 & $=0.5552$ \\
\hline $\begin{array}{l}\text { 2. What effect, if any, does the cytoskeleton have on exocytosis? What cytoskeletal } \\
\text { structure is involved in the function you described? }\end{array}$ & 0.22581 & 1.09677 & $<0.0001^{*}$ \\
\hline 3. Why does Tetrahymena pyriformis egest? & 1.1629 & 1.38710 & $=0.3220$ \\
\hline 4. Briefly describe the pathway a vesicle follows from ingestion to egestion. & 0.74194 & 1.70968 & $<0.0001^{*}$ \\
\hline $\begin{array}{l}\text { 5. What conclusions would you draw from the data shown below? (Students were shown } \\
\text { a hypothetical graph of average number of vesicles/cell over time [Supplemental } \\
\text { Material 2].) }\end{array}$ & 1.16129 & 0.87097 & $=0.2229$ \\
\hline
\end{tabular}

aSignificant differences were determined by a $t$ test. Scores were significantly higher after the experiment for question 2 ( $p<0.0001)$, and for question $4(p<0.0001)$. Questions 1,3 , and 5 did not show significantly higher scores after the assessment. Significant differences are marked with an* $(n=31)$.

exercise can be used individually or in conjunction with the previously described experiment. It may be used with given information (vesicle diameters, Tetrahymena width and length, and/or phagocytosis rates), as we did, or if time permits, the students can experimentally determine the dimensions of vesicles and Tetrahymena by measuring with an ocular micrometer. To determine phagocytic rate, students need to plot their data from the previously described experiment as a line graph. We used nonlinear regression analysis with Prism (GraphPad Software, La Jolla, CA) software to determine the initial slope. Alternatively, the slope of a best-fit line will give an approximation of the rate.

Using these values, the student calculates the surface area (SA) of Tetrahymena (a prolate spheroid) by SA = $2 \pi\left[a^{2}+a b(1.2)\right]$, where $a$ is the horizontal, transverse radius (given value: $a=12 \mu \mathrm{m}$ ) and $b$ is the vertical radius (given value: $b=28 \mu \mathrm{m}$ ). Vesicle SA is calculated by assuming vesicles are spherical and using the standard formula for the area of a sphere, $4 \pi r^{2}$ (given value: $r=2 \mu \mathrm{m}$ ). The rate of membrane turnover (RMT) is then determined by multiplying the initial rate of vesicle uptake by the vesicle SA. RMT can easily be compared among treatments as well.

In our exercise, students were asked to consider several investigative questions about maintaining the membrane SA. For example, if the plasma membrane is entering the cell by $20 \mu \mathrm{m}^{2} / \mathrm{min}$, what must be the rate of membrane addition by exocytosis to maintain a constant plasma membraneSA? Why is this exocytosis necessary? Finally, we asked the students to calculate how long it would take a cell to completely turn over its surface membrane, assuming the rate of phagocytosis is sustained.

To assess the effectiveness of this exercise, we asked students $(n=18)$ in our summer cell biology class to complete an assessment form composed of nine questions after completing this membrane turnover exercise (Supplemental Material $3)$. The students were asked to rank six statements on a scale of $1-5,1$ being "strongly disagree" and 5 being "strongly agree." In Table 2, we present the average score with SD for each question. The students agreed that this exercise helped them to visualize phagocytosis (mean $=4.2 \pm 0.9$ ), although the students were neutral about visualizing the process of exocytosis (mean $=2.9 \pm 1.2$ ). It must be noted that the students were assessed after a lab on phagocytosis only (Bozzone, 1999), not after the phagocytosis/exocytosis lab described here, so these

results were expected. The students were also somewhat neutral about visualizing how cellular processes work together (mean $=3.5 \pm 0.7$ ), although many students did agree with the statement (mode $=4$ ). The students agreed that the exercise helped them to apply mathematics to a biological system (mean $=4.4 \pm 0.5$ ), and most importantly, the students found this mathematical exercise to be somewhat interesting (mean $=3.3 \pm 1.0)$, and were not overwhelmingly frustrated (mean $=2.6 \pm 1.0$ ). In summary, students were able to understand the context of their phagocytosis/exocytosis rates and apply mathematics to a biological system in a relatively easy and straightforward manner.

In addition to responding to the six assessment statements, the students were asked to respond to three open-ended questions to determine what they felt was beneficial or not beneficial about the exercise. We also asked the students to tell us what the most surprising aspect of the exercise was (Supplemental Material 3). What follows are a few examples of their responses:
"The fact that it would take 200 minutes for Tetrahymena to completely internalize its membrane."
"It takes more than 2-3 hours for a tiny cell to turn over its membrane."

Table 2. Assessment of membrane turnover exercise ${ }^{\text {a }}$

Assessment statement

1. This exercise helped me to visualize the process of exocytosis.

2. This exercise helped me to visualize the process of phagocytosis.

3. This exercise helped me to visualize how cellular processes work together to carry out cell function.

4. This exercise helped me to apply math to a biological system.

5. This exercise was frustrating.

6. This exercise was interesting.

a Students $(n=18)$ were asked to rate the statements from 1 to 5 , 1 being "strongly disagree" and 5 being "strongly agree." Student opinion indicates that this exercise better enabled them to visualize phagocytosis and apply math to a biological system. 
"How simple the math was."

"The importance of exocytosis in preserving the integrity of the cell membrane."

"That the math actually has comprehensible applications."

The most beneficial aspects of the exercise according to the students were:

"Calculating the surface area and turnover number helped me to explain why exocytosis was useful."

"Step by step instructions on calculating RMT."

"The quantitative evidence of the membrane's ability to perform endocytosis (phagocytosis)."

"The very end of the exercise when we tied everything together (our rates, SA, etc)."

"Putting together the math. I'm a math minor, so it's always great when I get to bring math to biology."

Remarkably the students, found the entire exercise to be beneficial, as shown in the sampling of comments below when they were asked what was least beneficial:

"I think everything was beneficial because it all built up in the end, which helped me understand."

"I found every aspect beneficial."

The few students $(n=3)$ that did find something nonbeneficial did not entirely understand the exercise; an example of this type of comment follows:

"The SA of phagosomes. Did not understand the impact."

In summary, this demonstrates that the students did enjoy the exercise, and they found it beneficial, especially as an aid to the process of applying mathematics to biology.

\section{DISCUSSION}

Our phagocytosis/exocytosis assay was designed not only to help our students understand the processes of phagocytosis and exocytosis but also to expose them to a researchlevel experiment. Our results (and those of our students [unpublished data]) from this method of simultaneously measuring phagocytosis and exocytosis are very similar to those already published in the literature of experiments measuring either phagocytosis or exocytosis. Actin in Tetrahymena is present at the oral apparatus (site of phagocytosis; Nilsson, 1976) and the cytoproct (site of egestion; Allen and Wolf, 1979; Sugita et al., 2009), suggesting it is involved in both phagocytosis and exocytic egestion. Our data support this, demonstrating that actin disruption decreases the rates of both phagocytosis and exocytosis. Also of interest is a recent study by Sugita et al. (2009) showing that latrunculin-B (Lat-B) does not inhibit egestion. Reading this research paper provides ample opportunity for the students to delve into the primary literature. Discussions can be held after reading research papers to explore the differences between Lat-A and Lat-B and the differences between the experimental designs. Alternatively, there may be no difference; perhaps Lat-A does not decrease egestion directly, but indirectly. The processes of phagocytosis and exocytosis are intimately linked, and we know that Lat-A blocks phagocytosis, so there may not be enough vesicles with indigestible material to egest. Either way, exploring these issues not only gets the students deeper into the cellular mechanisms, but increases their knowledge of how to design experiments.

Studying microtubule function provides more chances for students to analyze and discuss the published literature. Our results indicate that microtubules are necessary for phagocytosis, most probably because microtubules are required for oral groove development (Nelson, 1970; Rothstein and Blum, 1974; Nilsson, 1976; Bozzone, 1999; Kovacs and Csaba, 2006). The most challenging aspect for students is to determine how microtubules are involved with exocytosis. It has been shown that microtubules are present at the cytoproct (Allen and Wolf, 1974, 1979; Kovacs and Csaba, 2006; Sugita et al., 2009); however, our results indicate that they are not necessary for egestion. Until recently, no one has reported on the function of microtubules during exocytic egestion. Sugita et al. (2009), with similar results to ours, indicate that egestion can still occur when microtubule action is blocked. Sugita and colleagues suggest that after egestion takes place, the recycled vesicles normally established by endocytosis cannot be formed, thereby blocking the cytoproct and preventing the vesicles that need to be egested from reaching the plasma membrane. It is startling that microtubules are not directly involved-or are they? Further research of the literature indicates that colchicine has a low affinity for Tetrahymena tubulin, as compared with other tubulins. Colchicine has also been shown to increase the synthesis of $\alpha$-tubulin (Stargell et al., 1992; Kovacs and Csaba, 2006). Thus, it is entirely conceivable that colchicine is ineffective in Tetrahymena. Discussion of this possibility provides students the opportunity to generate a new hypothesis and to develop additional experiments to test the role of microtubules in exocytic egestion.

After reading the literature and developing a working model of vesicle movement during the interconnected processes of phagocytosis and exocytosis, students are ready to ask their own questions in inquiry-based experiments. For example, our students have tested numerous variables, ranging from additional cytoskeletal drugs, chelators, and drugs that inhibit lysosomal function to altered $\mathrm{pH}$ and temperature.

We have established that the classroom experiment will provide data similar to data resulting from a research lab, but we must also demonstrate that the experiment is educational. To determine whether this experiment was successful at teaching exocytosis, we used a pre/post student-learning assessment. The results indicated that the students did indeed learn about exocytosis, although they did not learn the material presented in the prelab and were not able to perform data analysis. While it is clear that laboratory experiments are a beneficial tool for teaching science (Olson and LoucksHorsley, 2000; Gibson and Chase, 2002), it is apparent that the way this experiment was presented was not conducive to teaching skills in data analysis. Question 5, which asked the students to analyze hypothetical data, was presented such that the drug increased the rate of exocytosis, which is the opposite of both the logical hypothesis and the experimental results (Supplemental Material 2). Ultimately, the students performed more poorly on the postassessment data analysis 
question than on the preassessment, suggesting that they did not look at the graph but instead answered based on their preconceived notions and the results they had obtained in the experiment.

Our primary goal was to teach the processes of phagocytosis and exocytosis. Thus, in presenting the material and interpreting the data with the students, we focused on teaching content more than skill sets. While we ideally want every lab experience to teach scientific reasoning skills, this is not always possible, and in this situation it can be easily remedied in the subsequent inquiry-based laboratory sessions. Experiments such as ours, which include data analysis and, more specifically, experience in the creation and interpretation of graphs, have been shown to improve science literacy (Kitchen et al., 2003).

In an additional exercise that can serve several purposes, we had the students look at the rate of membrane turnover. This can be used to further critical thinking, by helping students to see how cellular processes are interconnected, as well as to introduce the students to the use of mathematics in biology. From our results, it is apparent that the students were able to take simple rates and vesicle numbers and apply the data to the cell itself. Ultimately, this is a straightforward and enjoyable method for teaching that mathematics is not only important, but crucial to understanding biological mechanisms.

This classroom experiment was designed to better introduce students to the concept of membrane dynamics, specifically phagocytosis and exocytosis. The purpose of this is not to just teach these crucial concepts but to set the stage for an optional second (or additional) week of inquiry-driven experiments. From the knowledge gained from this experiment, students are now better able to interpret their own experiments and describe what is happening at the molecular level.

In conclusion, this experiment provides ample opportunity to learn about the cellular processes of phagocytosis and exocytosis. Students learn the basics, as presented here, and there is potential to learn greater detail about the molecular mechanisms of phagocytosis, exocytosis, and the function of the cytoskeleton, as the students read the literature and develop their own researchable questions.

\section{ACCESSING MATERIALS}

For a copy of laboratory manual in PDF format, including background material and discussion questions, contact the corresponding author.

\section{ACKNOWLEDGMENTS}

We thank Mike Moran for the idea behind this project and the development of the Membrane Turnover Exercise. We thank the biology faculty of the University of Central Arkansas for constructive criticism of this work and Loren Stearman for his help with our statistical analysis. The project described was supported by award number P20RR16460 from the National Center for Research Resources. The content is solely the responsibility of the authors and does not necessarily represent the official views of the National Center for Research Resources or the National Institutes of Health.

\section{REFERENCES}

Alberts B, Johnson A, Lewis J, Raff M, Roberts K, Walter P (2008). Molecular Biology of the Cell, 5th ed., New York: Garland Science, pp. 787,800 .

Allen RD, Wolf RW (1974). The cytoproct of Paramecium caudatum: structure and function, microtubules, and fate of food vacuole membranes. J Cell Sci 14, 611-631.

Allen RD, Wolf RW (1979). Membrane recycling at the cytoproct of Tetrahymena. J Cell Sci 35, 217-227.

Blum JJ, Greenside H (1976). Particle ejection from the cytoproct of Tetrahymena. J Protozool 23, 500-502.

Bozzone DM (1999). Investigating phagocytosis in Tetrahymena. An experimental system suitable for introductory and advanced instruction. Am Biol Teacher 62, 136-139.

Coué M, Brenner S, Spector I, Korn ED (1987). Inhibition of actin polymerization by latrunculin A. FEBS Lett 213, 316-318.

Felder R (1993). Reaching the second tier: learning and teaching styles in college science education. J Coll Sci Teach 23, 286-290.

Fok AK, Shockley BU (1985). Processing of digestive vacuoles in Tetrahymena and the effects of dichloroisoproterenol. J Protozool 32, $6-9$.

Gibson HL, Chase C (2002). Longitudinal impact of an inquiry-based science program on middle school students' attitudes toward science. Sci Educ 86, 693-705.

Kitchen E, Bell JD, Reeve S, Sudweeks RR, Bradshaw WS (2003). Teaching cell biology in the large-enrollment classroom: methods to promote analytical thinking and assessment of their effectiveness. Cell Biol Educ 2, 180-194.

Kovacs P, Csaba G (2006). Effect of drugs affecting microtubular assembly on microtubules, phospholipid synthesis and physiological indices (signalling, growth, motility and phagocytosis) in Tetrahymena pyriformis. Cell Biochem Funct 24, 419-429.

Morton WM, Ayscough KR, McLaughlin PJ (2000). Latrunculin alters the actin-monomer subunit interface to prevent polymerization. Nat Cell Biol 2, 376-378.

Nelson ME (1970). Division delays and abnormal oral development produced by colchicine in Tetrahymena. J Exp Zool 175, 69-83.

Nilsson JR (1976). Physiological and structural studies on T. pyriformis GL. CR Trav Lab Carlsberg 40, 215-355.

Nilsson JR (1977). On food vacuoles in Tetrahymena pyriformis GL. J Protozool 24, 502-507.

Olson S, Loucks-Horsley S (2000). Inquiry and the National Science Education Standards: A Guide for Teaching and Learning, Washington, DC: National Academies Press

Ricketts TR (1979). Temporal movement of digestive vacuoles in fed Tetrahymena pyriformis GL-9. Protoplasma 100, 317-322.

Rothstein TL, Blum JJ (1974). Lysosomal physiology in tetrahymena III. Pharmacological studies on acid hydrolase release and the ingestion and egestion of dimethylbenzanthracene particles. J Cell Biol 62, 845-859.

Stargell LA, Heruth DP, Gaertig J, Gorovsky MA (1992). Drugs affecting microtubule dynamics increase $\alpha$-tubulin mRNA accumulation via transcription in Tetrahymena thermophila. Mol Cell Biol 12, 14431450.

Sugita M, Nakano K, Sata M, Toyooka K, Numata O (2009). The roles of actin cytoskeleton and microtubules for membrane recycling of a food vacuole in Tetrahymena thermophila. Cell Motil Cytoskeleton 66, 371-377.

Tiedtke A, Hunseler P, Florin-Christenesen J, Florin-Christensen M (1988). Exocytosis, endocytosis and membrane recycling in Tetrahymena thermophila. J Cell Sci 89, 515-520. 